conducted for several other cell types such as cardiomyocytes, human dermal fibroblasts, keratinocytes and chondrocytes. Finally, the platelet secretome from a murine model of thrombospondin-1 (TSP-1) deficiency - a potent angiostatic and proinflammatory factor - was studied. Statistics were performed by one-way ANOVA followed by a Tukey's post-hoc.

Results Platelet releasate dose-dependently stimulates myoblast proliferation and temporally stimulates differentiation. Proteomics reveals that PDGF and VEGF ligands are present in higher concentrations for PAR-1- versus collagen- or thrombinstimulated platelet secretomes. Inhibiting these ligand binding sites dose-dependently inhibits myoblast proliferation and differentiation. We have shown here for the first time that muscle stem cell markers for ex vivo proliferation and cell fate lineage (Cyclin D1 and Scrib, respectively) are upregulated when exposed to platelet releasate. Furthermore, the translational aspect of platelet releasate to an in vivo model shows accelerated regeneration. We show novel data that optimised platelet releasate can stimulate cardiomyocyte, human dermal fibroblast and chondrocyte proliferation and differentiation. Furthermore, we report for the first time that depletion of TSP-1 from platelet releasate promotes a more potent myoblast proliferation compared to wild-type.

Conclusion Platelet releasate is a powerful stimulant in many tissues for angiogenesis and cellular proliferation. Tissue-specific optimisation of the platelet preparation method is a critical step in producing an efficient biomaterial for regenerative medicine. Customisation of platelet releasate such as depletion of TSP-1 opens a new avenue for optimised regeneration.

Conflict of interest None

\section{BS36 REDUCED INSULIN-LIKE GROWTH FACTOR-1 RECEPTOR EXPRESSION ENHANCES VASCULAR REPAIR AND REGENERATION IN WHOLE BODY INSULIN RESISTANCE}

${ }^{1}$ Andrew Walker*, ${ }^{2}$ Anshu Sengupta, ${ }^{3}$ Peysh Patel, ${ }^{4}$ Noman Ali, ${ }^{4}$ Ben Mercer, ${ }^{1}$ Thomas Slater, ${ }^{1}$ Michael Drozd, ${ }^{3}$ Nicole Watt, ${ }^{3}$ Nadira Yuldasheva, ${ }^{3}$ Katherine Paradine, ${ }^{3}$ Nele Warmke, ${ }^{5}$ Mark Kearney, ${ }^{5}$ Richard Cubbon. ' Leeds Institute of Cardiovascular and Metabolic Medicine, The University of Leeds; ${ }^{2}$ Leeds Teaching Hospitals; ${ }^{3}$ University of Leeds; ${ }^{4}$ Leeds Teaching Hospitals NHS Trust; ${ }^{5}$ LICAMM, University of Leeds

\subsection{6/heartjnl-2019-BCS.198}

Introduction Insulin resistance is an independent risk factor for cardiovascular disease. We have previously shown that the insulin-like growth factor-1 receptor (IGF-1R) is a negative regulator of insulin receptor (IR) signalling, by sequestering IR subunits in insulin resistant IR:IGF1R 'hybrid receptors'. By crossing IR haploinsufficient (IRKO) mice with IGF-1R haploinsufficient (IGF-1RKO) mice (producing "double knockout" or DKO mice), we have previously demonstrated rescue of the
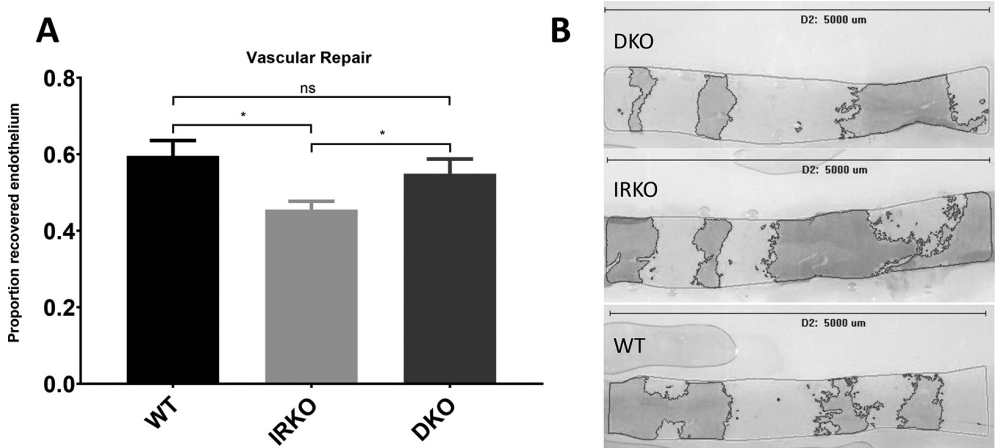

Abstract BS36 Figure 1 A) Vascular Repair The proportion of recovered endothelium was recorded 4 days following denuding femoral artery wire injury. N=10 (WT, 8 (IRKO), 14 (DKO); B) Vascular Repair Images Representative images of femoral arteries 4 days following denuding wire injury photographed at 20x magnification. Evans blue dye highlights subendothelial structures (blue), i.e. areas not yet re-endothelialised
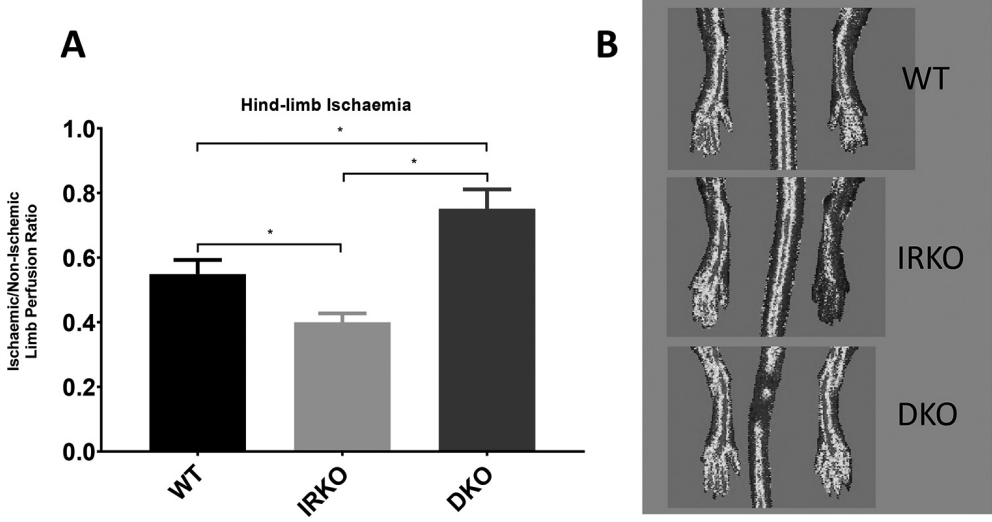

Abstract BS36 Figure 2 A) Vascular Regeneration Laser Doppler flux results at day 21 for each group. N=18 (WT), 19 (IRKO), 12(DKO). B) Vascular Regeneration Images Representative Laser Doppler flux images from top to bottom: wild type; IRKO; DKO. Right leg (left of image) has undergone sham procedure as control, left leg (right of image) has had induction of ischaemia with ligation and removal of femoral artery 21 days prior to imaging 
endothelial dysfunction caused by insulin resistance. Whether this is associated with wider benefits in vascular biology is unclear. Our original hypothesis is that reduced expression of the IGF-1R in whole body insulin resistance improves vascular repair and regeneration.

Methods Metabolic assessment included measurement of weight gain and glucose and insulin tolerance testing. Denuding femoral artery endothelial injury was induced with angioplasty guidewire and repair was quantified by Evans Blue perfusion 4 days later. For hindlimb ischemia, the left femoral artery was ligated and excised, with sham surgery contralaterally. Control:ischemic limb perfusion ratio was assessed with weekly laser Doppler imaging for 4 weeks. Data are expressed as mean (standard error) and compared using t-tests; * denotes $\mathrm{p}<0.05$; $\mathrm{ns}=$ not significant.

Results Glucose and insulin tolerance tests were similar in DKO and IRKO mice. Body weight was significantly lower in DKO than IRKO [area under curve (arbitrary units) 116(2.1) Vs $\left.123(2.4)^{*} \mathrm{n}=15\right]$. DKO had a significantly greater proportion of recovered endothelium (Figure $1 \mathrm{~A} \& \mathrm{~B}$ ) after denuding wire injury to the femoral artery compared with IRKO [0.55 (0.04) Vs 0.46 (0.02) p=0.047*, n=8-14]. ]. DKO had superior recovery (Figure $2 \mathrm{~A} \& \mathrm{~B}$ ) after induction of hindlimb ischemia [area under curve limb perfusion ratio (arbitrary units) 2.2(0.11) Vs 1.3(0.08)* $\mathrm{n}=12-19]$.

Conclusion Reduced IGF-1R expression improves vascular repair and regeneration in the context of whole-body insulin resistance. Further work will aim to elucidate the possible mechanisms for these novel observations.

Conflict of interest None

\section{BS37 UNDERSTANDING THE GERMINAL CENTRE B CELL RESPONSE TO ATHEROSCLEROSIS IN MICE USING LINEAGE TRACING}

Anne Francis, Andrew Sage. University of Cambridge

\subsection{6/heartjnl-2019-BCS.199}

Introduction Elevated plasma low density lipoprotein (LDL) is a major risk factor for atherosclerosis and its immunogenic oxidation triggers an inflammatory response. The importance of $\mathrm{B}$ cells within cardiovascular disease is demonstrated by genome-wide association studies and transcriptomic studies that have identified genes involved in proliferation and activation of $\mathrm{B}$ cells. It has been shown that the germinal centre (GC) response, the process by which plasma and memory B cells are formed, is pathogenically dysregulated in atherosclerosis, and that class-switched plasma cells infiltrate into human diseased vascular tissue. We therefore sought to further characterise and understand the causes for this pathogenic response by using a lineage tracing mouse model.

Methods Use of the tamoxifen-inducible AID-CreERT2-RosaEYFP-Ldlr-/- lineage tracing mouse model enables the tracking of atherosclerosis-specific B cell clones comprising GC, memory and plasma B cells. Ldlr-/- and Ldlr \pm ('WT') mice were fed chow or western diet (WD) for up to 8 weeks and upon tamoxifen dosing via intra-peritoneal injection, AID-expressing cells (GC B cells) are fluorescently labelled with EYFP. The timing of tamoxifen dosing was varied throughout the studies. Results Ldlr-/- mice develop an enlarged GC response within the spleen and lymph nodes, although not in gut-associated Peyer's patches. Around $50 \%$ of Ldlr-/- mice on chow diet develop this response whereas $>80 \%$ of WD-fed mice have an enhanced response. WT mice do not develop this response. The GC response consists of more class-switched (IgM-) GC B cells in Ldlr-/- mice compared to WT mice. This results in increased serum levels of the Th1-driven $\operatorname{IgG}$ isotype $\operatorname{IgG} 2 \mathrm{c}$, but no increase in Th2-driven IgG1 after 8 weeks western diet. Labelling GC cells during western diet (but not before) demonstrates that GC clones persist longer after western diet feeding than in chow-fed mice.

Conclusion Atherosclerotic conditions increase not only numbers of GC B cells, but also change the type of response that occurs, providing an opportunity to target the atherosclerosisspecific responses therapeutically.

Conflict of interest None

\section{BS38 ROLE OF NEUTROPHIL ELASTASE IN ABDOMINAL AORTIC ANEURYSMS AND THORACIC AORTIC DISSECTION}

Stuart Pearce*, Qingzhong Xiao, Wei Wu, WeiWei An, Mei Yang. Barts and The London, Queen Mary

\subsection{6/heartjnl-2019-BCS.200}

Abdominal Aortic Aneurysm (AAA) affects 4-5\% of men over 65, and Thoracic Aortic Dissection (TAD) is a life-threatening aortic pathology where $75 \%$ of patients die within 2 -weeks post-onset. Relatively little is known about the underlying mechanisms, which warrants further investigation. Neutrophil Elastase (NE) is an enzyme with roles in priming of the immune system, clearance of large pathogens and remodelling of extra-cellular-matrix proteins, all influential in AAA and TAD. Our recent study suggests a causal role for $\mathrm{NE}$ in hyperlipidemia-induced atherosclerosis. However, little is known regarding implications of NE in AAA and TAD. This Study aims to investigate the role of NE within both pathologies.

Gene-expression of NE and AAA-associated markers, MMP2 and MMP-9, were significantly up-regulated by $\mathrm{CaCl} 2$-and AngII-treatment in the cultured vascular smooth muscle cells, endothelial cells and macrophages. In both AngII- and $\mathrm{CaCl} 2$ induced AAA mouse models, reduction of aortic expansion in NE-knockout mice was observed, compared with wild-type littermates. TAD experiments reaffirmed the functional importance of NE, with significant reduction in death within NEknockout mice. Histological and Proteomics analysis was carried out in order to determine changes produced by loss of the NE gene within these models. Preliminary translational work is underway, with an Audit of Aneurysm patients' blood profiles. Additionally, peripheral blood and aortic tissues were harvested from surgical repair patients with AAA for NE expression analysis. Initial results show an alteration in proportions of White Blood cell populations, namely macrophages 